\title{
Comparison of tamsulosin vs tamsulosin/sildenafil effectiveness in the treatment of erectile dysfunction in patients affected by type III chronic prostatitis
}

\author{
Ubaldo Cantoro, Francesco Catanzariti, Vito Lacetera, Luigi Quaresima, \\ Giovanni Muzzonigro, Massimo Polito \\ Institute of Urology, Polytechnic University of Marche, Azienda O.U. Ospedali Riuniti, Ancona, Italy.
}

\begin{abstract}
Aim: We evaluated the effectiveness of tamsulosin monotherapy versus tamsulosin plus Summary sildenafil combination therapy on erectile dysfunction (ED) in young patients with type III chronic prostatitis and ED by using symptom score scales.

Materials and methods: 44 male patients were divided into 2 groups: the first group (20 patients) was treated with tamsulosin 0,4 $\mathrm{mg}$ monotherapy and the second one (24 patients) was treated with tamsulosin $0,4 \mathrm{mg}$ plus sildenafil $50 \mathrm{mg}$ combination therapy. "International Prostate Symptom Score" (IPSS), "National Institute of Health Chronic Prostatitis Symptom Index" (NIH-CPSI) and "International Index of Erectile Function" (IIEF-5) were investigated in each group of patients, and scores calculated during the first medical examination. Both groups were treated with tamsulosin once daily for 60 days, while sildenafil $50 \mathrm{mg}$ was given on demand (at least 2 times per week) for 60 days. During the second medical examination IPSS, NIH-CPSI and IIEF-5 scores were analyzed once more. Afterwards, the alterations of scores among medical examinations in each group and between both groups were statistically compared. Results: The age average of the 44 cases included was $32.04 \pm 3.15$ years. Both groups present a statistically significant decrease, between the first and the second medical examination, in IPSS, NIH-CPSI scores and statistically significant increase in IIEF-5 score. In addition, there is no statistically significant difference, in all scores, between mono and combination therapy. Conclusions: tamsulosin monotherapy, as well as a combination therapy (tamsulosin plus sildenafil) has an improving effect on symptoms and on ED in patients with type III prostatitis. In the near future alpha-blockers monotherapy could be used in the treatment of chronic prostatitis and ED cases instead of phosphodiesterase type 5 (PDE-5) inhibitors combination therapy.
\end{abstract}

KEY WORDS: Chronic prostatitis; Erectile dysfunction; Tamsulosin; Sildenafil.

\section{INTRODUCTION}

The aim of this study is to evaluate the effectiveness of tamsulosin in patients affected by Low Urinary Tract Symptoms (LUTS) and erectile dysfunction (ED), and also to compare this monotherapy with one combined with sildenafil, belonging to phosphodiesterase type 5 (PDE-5) inhibitors drug class, which are the recommended first-line treatment for ED.

Type III chronic prostatitis or Chronic prostatitis/chronic pelvic pain syndrome (CP/CPPS) is characterized by abdominal, pelvic, genital pain, obstructive or irritative LUTS and by the absence of urinary tract infection (1). Many studies showed its association with painful premature ejaculation and with erectile dysfunction. CP/CPPS occurs frequently in young patients and is one of the organic causes of erectile dysfunction (ED) in this age range. Therefore, a common pathogenic mechanism for these two diseases is likely to exist (2).

Adult-old patients LUTS affected have two times higher 
risk to develop ED, since the prevalence of LUTS is of $72.2 \%$ in males affected also by ED and of $37.7 \%$ in males with no ED (3)

Literature data show that ED associates with the severity of LUTS but, although studies pointed out a correlation between CP/CPPS and ED, they do not provide with any explication of pathogenic mechanisms (4).

Many pathogenic mechanism were investigated to find an explanation to ED in young patients affected by $\mathrm{CP} / \mathrm{CPPS}$. Any connection with hypogonadism neither other endocrine disorders were found, except for a study which, unlike controls, found higher levels of testosterone in patients with CP/CPPS (5).

Another study found an association with hypogonadism, due to the fact that patients took opioids for long periods because of LUTS severity (6). Vascular diseases and arterial insufficiency are well known causes of ED, even though they are uncommon in young patients (7). Anyway, one study pointed out alterations in the peripheral arterial tone in patients with CP/CPPS (8), due to a endothelial vascular dysfunction mediated by nitric oxide (9).

Moreover, the arterial flow can be compromised from the outside by spastic contractions of pelvic floor (10). It is known muscle relaxant therapies can have positive effects on ED (11).

Occlusive vessel disease is a condition which frequently occurs in old patients, also in presence of penile fibrosis. Therefore, also this pathogenic mechanism is uncommon in young patients. Although ED psychogenic cause was not adequately investigated in patients with CP/CPPS, a relation may exist since often patients affected by painful syndromes also suffer from stress, anxiety and maladaptive responses to stressful events ("catastrophizing") (12).

\section{MATERIALS AND METHOdS}

Our study analyzed a number of 44 patients who were examined at our Clinic because affected by type III chronic prostatitis associated with erectile dysfunction since at least 6 months. All patients were sexually active. We excluded from the study all patients affected by infections of the urinary system, neoplasia, congenital disorders, previous surgeries, urolithiasis and hyperactive bladder. None of the included patients used PDE-5 in the past. None of the examined patients presented side effects due to the use of alpha-blockers and PDE-5. Patients were examined through anamnesis, which is a clinical exam with neurological evaluation of the pelvic floor and rectal examination, uroflowmetry, suprapubic ultrasound evaluation of post-void residual, trans-rectal prostate ultrasound, total PSA, microscopic and cultural exams of urine and semen and urethral secretion after prostate massage. The 44 patients were divided into 2 groups: the first group (20 patients) was treated with monotherapy, tamsulosin $0.4 \mathrm{mg}$, the second one ( 24 patients) was treated with a combination therapy, tamsulosin $0.4 \mathrm{mg}$ plus sildenafil $50 \mathrm{mg}$. Patients assignment to one group or the other was random. The average age of patients included in the study is $32.04 \pm 3.15$ years. None of patients was affected by BPH; prostate volume range was between 15 and $25 \mathrm{ml}$. Both uroflowmetry parameters and post-void residuals were not pathological.
During the first medical examination, all patients were subjected to "International Prostate Symptom Score" (IPSS), "National Institute of Health Chronic Prostatitis Symptom Index" (NIH-CPSI) and "International Index of Erectile Function" (IIEF-5). Both groups were treated with tamsulosin for 60 days; sildenafil $50 \mathrm{mg}$ was taken when needed before a sexual intercourse (at least 2 times per week) and for 60 days by the second group. During the second medical examination, 60 days later, all patients were subjected again to IPSS, NIH-CPSI and IIEF-5.

We considered mild patients' symptoms with IPSS score between 0-7 and NIH-CPSI between 0-14; moderate respectively between 8-19 and 15-29 and severe between 20-35 and $>30$. We considered mild patients' erectile dysfunction with IIEF-5 score between 17-21, mildmoderate between 12-16, moderate between 8-11 and severe between 5-7.

We statistically evaluated a potential difference in IIEF-5 scores according to the symptomatic severity of IPSS and NIH-CPSI and in the last two questionnaires scores according to IIEF-5 severity.

Therefore, we statistically evaluated the differences of questionnaires scores means between the two medical examinations in each group and between the two groups. For the statistic analysis we used Graphpad Prism 5 program. In addition to the descriptive statistic modes (mean, standard deviation), oneway ANOVA, KruskalWallis, Mann-Whitney and Student $t$ test were used for a statistic evaluation. The results were analyzed with a significance level of $\mathrm{P}<0.05$.

\section{RESULTS}

Table 1 shows the mean of questionnaires analyzed and the mean of patients' figures. According to IPSS questionnaire, 4 patients presented mild symptoms, 26 moderates and 14 severe; according to NIH-CPSI questionnaire 6 patients presented mild symptoms, 29 moderates, 9 severe; according to IIEF- 5 questionnaire 8 patients suffered from mild erectile dysfunction, 17 mild-moderate, 13 moderate, 6 severe. We confronted IIEF-5 score means of patients with mild, moderate and severe symptoms according to IPSS and we did not notice any statistically difference: ANOVA $(\mathrm{p}=0,87)$ and Kruskal-Wallis $(\mathrm{p}=0.92)($ Table 2$)$.

Moreover, there is no statistically difference between IPSS

Table 1.

General characteristics and mean symptom scores of the cases.

\begin{tabular}{|lccc|}
\hline & Mean & SD & Range \\
\hline Age $($ year $)$ & 32.04 & 3.15 & $23-35$ \\
\hline $\mathbf{P V ~}(\mathrm{ml})$ & 17.20 & 2.56 & $15-25$ \\
\hline $\mathbf{Q}_{\max }(\mathrm{ml} / \mathrm{s})$ & 21.24 & 3.45 & $17.8-28.3$ \\
\hline PMR $(\mathrm{ml})$ & 27.18 & 8.78 & $0-42$ \\
\hline IPSS & 13.52 & 1.49 & $8-24$ \\
\hline IPSS-QOL & 3.87 & 0.27 & $0-5$ \\
\hline IIEF-5 & 12.41 & 0.66 & 5.21 \\
\hline NIH-CPSI & 17.51 & 1.92 & $5-28$ \\
\hline
\end{tabular}


Table 2.

The effect of IPSS level on mean IIEF-5 score.

\begin{tabular}{|lcl|}
\hline \multicolumn{3}{|c|}{ Mean IIEF-5 score } \\
\hline All cases & $12.41 \pm 0.66$ & \\
\hline IPSS & $13.28 \pm 0.89$ & ANOVA $P=0.87$ \\
Mild & $12.85 \pm 0.39$ & Kruskal-Wallis $\mathrm{P}=0.92$ \\
Moderate & $12.13 \pm 1.98$ & \\
Severe &
\end{tabular}

Table 3.

The effect of IIEF-5 level on mean IPSS score.

\begin{tabular}{|lcl|}
\hline \multicolumn{3}{|c|}{ Mean IPSS score } \\
\hline All cases & $13.52 \pm 1.49$ & \\
\hline IIEF-5 & & \\
Mild & $13.25 \pm 1.17$ & ANOVA P $=0.43$ \\
Mild-moderate & $14.48 \pm 0.96$ & Kruskal-Wallis $P=0.61$ \\
Moderate & $14.81 \pm 0.91$ & \\
Severe & $12.84 \pm 1.57$ & \\
\hline
\end{tabular}

Table 4.

The effect of NIH-CPSI level on mean IIEF-5 score.

\begin{tabular}{|lcl|}
\hline \multicolumn{3}{|c|}{ Mean IIEF-5 score } \\
\hline All cases & $12.41 \pm 0.66$ & \\
\hline NIH-CPSI & \\
Mild & $14.28 \pm 0.27$ & ANOVA $P=0.12$ \\
Moderate & $12.05 \pm 0.39$ & Kruskal-Wallis $P=0.25$ \\
Severe & $15.13 \pm 1.16$ & \\
\hline
\end{tabular}

Table 5.

The effect of IIEF-5 level on mean NIH-CPSI score.

\begin{tabular}{|lcl|}
\hline \multicolumn{3}{|c|}{ NIH-CPSI score } \\
\hline All cases & $17.51 \pm 1.92$ & \\
\hline IIEF-5 & \\
Mild & $22.92 \pm 0.87$ & ANOVA P $=0.18$ \\
Mild-moderate & $19.08 \pm 0.56$ & Kruskal-Wallis $\mathrm{P}=0.26$ \\
Moderate & $17.83 \pm 0.74$ & \\
Severe & $21.98 \pm 1.19$ & \\
\hline
\end{tabular}

score means of patients with mild, mild-moderate, moderate, severe erectile dysfunction: ANOVA $(p=0,43)$ and Kruskal-Wallis ( $\mathrm{p}=0,61$ ) (Table 3 ). We did not notice any statistically difference even through the comparison of IIEF-5 scores according to NIH-CPSI mild, moderate and severe symptoms: ANOVA ( $\mathrm{p}=0,12)$, Kruskal-Wallis $(\mathrm{p}=0,25)$ (Table 4); any difference also in NIH-CPSI scores according to IIEF-5: ANOVA $(p=0,18)$, Kruskal-Wllis $(\mathrm{p}=0,26)$ (Table 5).

We noticed, inside each therapy group, a statistically relevant decrease, between the first medical examination and 60 days later, in IPSS, IPSS-QOL and NIH-CPSI score. We also pointed out a statistically relevant increase in IIEF-5 score (Table 6 -7). We did not notice, in 60 days, a statistically relevant difference, between the two therapy groups, in all questionnaires score, IIEF-5 included (Table 8).

Table 6.

Alterations in IPSS, IIEF-5, NIH-CPSI, IPSS-QOL between visit 1 and visit 2 in Group 1 treated with tamsulosin 0,4 mg.

\begin{tabular}{|lccc|}
\hline & Visit 1 & Visit 2 & P \\
\hline IPSS & $13.26 \pm 0.92$ & $8.23 \pm 0.72$ & $<0.001$ \\
\hline IIEF-5 & $12.54 \pm 0.59$ & $17.83 \pm 1.46$ & $<0.001$ \\
\hline NIH-CPSI & $17.87 \pm 1.14$ & $10.54 \pm 1.35$ & $<0.001$ \\
\hline IPSS-QOL & $3.95 \pm 0.22$ & $2.02 \pm 0.56$ & $<0.001$ \\
\hline
\end{tabular}

Table 7.

Alterations in IPSS, IIEF-5, NIH-CPSI, IPSS-QOL between visit 1 and visit 2 in Group 2 treated with tamsulosin 0,4 mg plus sildenafil $50 \mathrm{mg}$.

\begin{tabular}{|lccc|}
\hline & Visit 1 & Visit 2 & P \\
\hline IPSS & $13.75 \pm 1.84$ & $8.07 \pm 0.91$ & $<0.001$ \\
\hline IIEF-5 & $12.31 \pm 0.78$ & $18.75 \pm 1.24$ & $<0.001$ \\
\hline NIH-CPSI & $17.47 \pm 2.09$ & $9.74 \pm 1.98$ & $<0.001$ \\
\hline IPSS-Q0L & $3.65 \pm 0.41$ & $1.82 \pm 0.25$ & $<0.001$ \\
\hline
\end{tabular}

Table 8.

Alterations in IPSS, IIEF-5, NIH-CPSI, IPSS-QOL between Group 1 and Group 2 after 60 days.

\begin{tabular}{|lccc|}
\hline & Group 1 & Group 2 & P \\
\hline IPSS & $8.23 \pm 0.72$ & $8.07 \pm 0.91$ & 0.751 \\
\hline IIEF-5 & $17.83 \pm 1.46$ & $18.75 \pm 1.24$ & 0.835 \\
\hline NIH-CPSI & $10.54 \pm 1.35$ & $9.74 \pm 1.98$ & 0.486 \\
\hline IPSS-QOL & $2.02 \pm 0.56$ & $1.82 \pm 0.25$ & 0.574 \\
\hline
\end{tabular}

\section{Discussion}

Chronic prostatitis/chronic pelvic pain syndrome (CP/CPPS) is a syndrome characterized by pain (abdominal, pelvic, genital), obstructive and irritative LUTS in absence of infection (1), causing Quality Of life (QOL) decrease (13).

Although it is known that type III chronic prostate associates with erectile dysfunction, it is still less clear the etiophatogenesis implied in these two nosological entities. Great part of clinical studies examine old patients with LUTS and ED, but also with concomitant BPH and obstruction of urine flow. Still few are studies which investigate the presence of ED in younger patients affected by type III chronic prostatitis in absence of BPH and obstruction.

The most supported theory explaining the common pathogenic mechanism of LUTS and concomitant ED, independently from $\mathrm{BPH}$, points out there is a hyperactivity of autonomic nervous system and endothelial alterations due to the effects on nitric oxide - cyclic 
monophosphate guanosine e alterations in Rho-kinase pathway (14).

Some studies already evaluated the effectiveness of alpha-blockers in treating erectile dysfunction associated with LUTS (15-16), but it was not compared with PDE5 inhibitors and, as stated above, the patients examined were old people with concomitant $\mathrm{BPH}$.

In our study, after 60 days of therapy, each group showed statistically relevant improvements in questionnaires scores: IPSS, IPSS-QOL, IIEF-5, NIH-CPSI.

In other words, tamsulosin, as well as the combination therapy of tamsulosin and sildenafil, improved both LUTS and ED. Sixty days after the therapy, we did not stress out any statistically relevant difference in questionnaires scores between the two groups, despite a PDE- 5 inhibitors treatment in the second group. Moreover, there is no correlation between LUTS severity (classified according to IPSS and NIH-CPSI), ED rate and vice versa. These results suggest tamsulosin may improve ED and chronic prostatitis symptoms, reducing the spasm of prostate smooth muscle, the associated inflammation and improving prostate and penis blood flow.

Anyway, our study has several limitations; it does not have a placebo control arm and it is circumscribed. We are also persuaded other studies are needed for evaluating monotherapy and combination therapy for a longer period than 60 days. Moreover, patients' randomization does not guarantee a complete randomness: patients' assignment to one study group or the other was made through their alternated insertion in one of the two groups.

\section{Conclusions}

LUTS severity in young patients suffering from type III chronic prostatitis does not correlate with the severity of ED and vice versa. Tamsulosin therapy for the treatment of young patients with type III chronic prostatitis together with erectile dysfunction has the same effectiveness of the most expensive combination therapy (tamsulosin and sildenafil). We are persuaded in the future the cheaper therapy with alpha-blocker will be used in CP/CPPS and ED affected patients.

\section{REFERENCES}

1. Schaeffer AJ, Datta NS, Fowler JEJ, et al. Overview summary statement. Diagnosis and management of chronic prostatitis/chronic pelvic pain syndrome (CP/CPPS). Urology. 2002; 60:1-4.

2. Costabile RA, Steers WD. How can we best characterize the relationship between erectile dysfunction and benign prostatic hyperplasia? J Sex Med. 226; 3:676-681.

3. Carson CC. Combination of phosphodiesterase-5 inhibitors and alpha-blockers in patients with benign prostatic hyperplasia: treatments of lower urinary tract symptoms, erectile dysfunction, or both? BJU Int. 2006; 97:39-43

4. Shiri R, Hakkinen JT, Hakama M, et al. Effect of lower urinary tract symptoms on the incidence of erectile dysfunction. J Urol. 2005; 174:205-209.

5. Dimitrakov J, Joffe HV, Soldin SJ, et al. Adrenocortical hormone abnormalities in men with chronic prostatitis/chronic pelvic pain syndrome. Urology. 2008; 71:261-6.
6. Daniell HW. Hypogonadism in men consuming sustained-action oral opioids. J Pain. 2002; 3:377-84.

7. Gonen M, Kalkan M, Cenker A, et al. Prevalence of premature ejaculation in Turkish men with chronic pelvic pain syndrome. J Androl. 2005; 26:601-3.

8. Shoskes DA, Prots D, Karns J, et al. Greater endothelial dysfunction and arterial stiffness in men with chronic prostatitis/chronic pelvic pain syndrome-a possible link to cardiovascular disease. J Urol. 2011; 186:907-10.

9. Rubinshtein R, Kuvin JT, Soffler M, et al. Assessment of endothelial function by non-invasive peripheral arterial tonometry predicts late cardiovascular adverse events. Eur Heart J. 2010; 31:1142-8.

10. Shoskes DA, Berger R, Elmi A, et al. Muscle tenderness in men with chronic prostatitis/chronic pelvic pain syndrome: the chronic prostatitis cohort study. J Urol. 2008; 179:556-60.

11. Anderson RU, Wise D, Sawyer T, et al. Sexual dysfunction in men with chronic prostatitis/chronic pelvic pain syndrome: improvement after trigger point release and paradoxical relaxation training. J Urol. 2006; 176:1534-8.

12. Nickel JC, Tripp DA, Chuai S, et al. Psychosocial variables affect the quality of life of men diagnosed with chronic prostatitis/chronic pelvic pain syndrome. BJU Int. 2008; 101:59-64.

13. McNaughton Collins M, Pontari MA, O'Leary MP, et al. Quality of life is impaired in men with chronic prostatitis: the Chronic Prostatitis Collaborative Research Network. J Gen Intern Med. 2001; 16:656-62.

14. Rosen RC, Wei JT, Althof SE, et al. Association of sexual dysfunction with lower urinary tract symptoms of BPH and BPH medical therapies: results from the BPH registry. Urology. 2009; 73:562-566.

15. Kirby RS, Andersen M, Gratzke P, et al. A combined analysis of double-blind trials of the efficacy and tolerability of doxazosin-gastrointestinal therapeutic system, doxazosin standard and placebo in patients with benign prostatic hyperplasia. BJU Int. 2001; 87:192-200.

16. De Rose AF, Carmignani $G$, Corbu $C$, et al. Observational multicentric trial performed with doxazosin: evaluation of sexual effects on patients with diagnosed benign prostatic hyperplasia. Urol Int. 2002; 68:95-98

\section{Correspondence}

Ubaldo Cantoro, MD (Corresponding Author)

Resident in Urology

ubaldocantoro@tiscali.it

Francesco Catanzariti, MD

Resident in Urology

fracatanzariti@libero.it

Vito Lacetera, MD

Urologist, Resident in Urology

vlacetera@gmail.com

Luigi Quaresima, MD

Resident in Urology

luigiquaresima@yahoo.it

Giovanni Muzzonigro MD

Professor of Urology and Chief Institute of Urology, Resident in Urology g.muzzonigro@univpm.it

Massimo Polito, MD

Urologist, Resident in Urology

max_polito@virgilio.it

Institute of Urology, A.O. Ospedali Riuniti

Via Conca 71 - I-60020 Ancona, Italy 